\title{
Free Vibration Analysis of Punctured Plate by Matching Polynomials and Constraint Technique
}

\author{
Sasank Sekhar Hota \\ Department of Civil Engineering, SIET, Dhenkanal-759001, India \\ Mrutyunjay Rout \\ Department of Mechanical Engineering, GCE, Kalahandi-766001, India \\ Payodhar Padhi \\ Department of Mechanical Engineering, KIST, Bhubaneswar-752050, India
}

(Received 27 July 2009, revised 26 April 2010, accepted 1 November 2010)

The hallmark of this paper is the adoption of the constraint technique, in combination with a subparametric, triangular-plate bending element of first-order shear deformation, to maintain uniform mesh size and shape even while dealing with cutouts of arbitrary shapes. The evolution of two cutout models in the present investigation is a distinct improvement over the existing practices of cutout analysis. The use of matching polynomials offers the scope of eliminating the hazards of locking and spurious zero energy modes, while solving problems of very thin plates. Benchmark examples, as well as the author's own problems on free vibration of rectangular plates with different shapes of cutouts, have been solved to exhibit versatility of these models. Mode shapes of plates with different shapes of cutout have also been provided.

\section{NOMENCLATURE}

$\begin{array}{ll}a, b & \text { length and width of plate } \\ E & \text { Young's modulus } \\ h & \text { Plate thickness } \\ v & \text { Poisson's ratio } \\ \rho & \text { mass density of plate } \\ \varpi & \text { Nondimensional fundamental frequency } \\ & \left(=\omega b^{2}\left[12 \rho\left(1-v^{2}\right) / E h^{2}\right]^{1 / 2}\right)\end{array}$

\section{INTRODUCTION}

Study on cutouts is usually used in engineering as well as in biomechanics. $^{1,2}$ Researchers' attention has been attracted to the analysis of punctured skin since the mid-1940s. After the pioneering work of Lure, ${ }^{3}$ research in this area flowed down in the form of experiments and theoretical analyses. ${ }^{4,5}$

Functional and even design requirements demand provision of cutouts in plate and shell structures. ${ }^{6}$ Edges of cutouts are made curved to improve aesthetics and to reduce stress concentration. Punctures of irregular shapes appear in structural elements due to damage during their service life. ${ }^{7}$ All these reasons offer justification for the proposition of putting forward methods to deal with arbitrarily shaped cutouts, which are available in semianalytical and purely computational forms. ${ }^{7,8}$

Analysis of a punctured plate/shell is usually conducted by providing fine meshing near the discontinuity. Computer codes for such an approach cannot be called a general purpose programme because the discretization shape, number, and overall meshing pattern become unique for each case. The number of plate or shape elements not only depends on the size of the cutout but also on its shape, and even on its location in the structure. Moreover as the element shapes and sizes throughout the structure do not remain uniform, the use of identical plate elements for computation of element matrices is not possible. Thus, these methods increase the cost of the generation of global matrices because of repetitive computation. To be more clear, consider the case of a rectangular cutout. The mesh divisions towards the cutout boundary become finer, 6,9 thereby causing an increase in the total number of elements, which would have been much less, had there been no cutout. Some of the publications even make no mention of the method they adopt for modeling the cutouts. ${ }^{10-16}$ Commercial packages available in the market usually generate quadrilaterals of different sizes near the cutout, with mesh lines aligned around the cutout boundary. ${ }^{17-19}$ This method of aligning the mesh lines around the cutout boundary not only increases the total degrees of freedom, but also raises a question about the uniformity in the level of calculation precision of the stiffness and the mass matrices. This is because, for example, the investigators have integrated the element matrices by reduced integration to get rid of shear locking. ${ }^{20,21}$ Though this may give some degree of accuracy to the element matrices of the rectangular elements (away from the cutout), the characteristic matrices of the quadrilateral elements (near the cutouts), being fractions of polynomials, would need more Gauss points to achieve the same degree of accuracy. Moreover, the other deficiency of these elements not being compact is also inherent in them. These problems become more pronounced if an arbitrarily shaped perforation is encountered, in case of which a good number of quadrilaterals and triangles will have to surround the cutout.

All these shortcomings can be eliminated if the plate can be discretized into a number of square and compact elements, notwithstanding the shape and size of the hole. This can be achieved if the portions of the cutout, obtained by the process of routine discretization of the plate, are treated as slave ele- 